\title{
Measurement System of Body Conduction Sounds Using a Mbed Microcontroller
}

\author{
Fumiaki Ehara ${ }^{\mathrm{a}, *}$, Takahiro Kubo ${ }^{\mathrm{b}}$, Kanya Tanaka ${ }^{\mathrm{b}}$ \\ a Ube National College of Technology, 2-14-1 Tokiwadai, Ube-shi, Yamaguchi 755-8611, Japan \\ byamaguchi University, 2-16-1 Tokiwadai, Ube-shi, Yamaguchi 755-8611, Japan \\ *Corresponding Author: ehara@ube-k.ac.jp
}

\begin{abstract}
Solitary death of elderly people is recognized as social problem in Japan. There is a real need for a new device to monitor the health condition of elderly people. In this study, we made a measurement system of body conduction sounds using a body conduction sound sensor, mbed LPC1768 and XBee. A body conduction sound sensor using electret condenser microphone is filled up with polyurethane resin. The mbed LPC1768 has a 32 bit microcontroller, it has more coping skills than the other 8 bit microcontrollers and it is good to deal with sound data. The system can communicate to a computer over a radio using XBee. It can filter out the noise using a median filter. We measured several body conduction sounds using this system at the cardiac apex, neck and wrist. We could measure coronary sounds at the cardiac apex well, and could measure arterial sounds and the pulse waves at the neck and wrist well.
\end{abstract}

Keywords: body conduction sound, electrets condenser microphone, mbed.

\section{Introduction}

Solitary death of elderly people is recognized as social problem in Japan. It is difficult to substantiate a system which monitor them using camera because of lack of privacy. There is a real need for a new monitoring system that conscious of privacy. A body conduction sound that produced and propagated inside of the body is measured by a sensor that is put on the body surface. The sound is used to find an abnormality in medicine field. The doctor listens to the sound through a stethoscope, and uses it to discovery of the illness. Some researchers reported a system to find unusual sounds of the heart ${ }^{(1,2)}$, and an analysis method was reported that finds an abnormal cardiac sound measured by the wireless electronic stethoscope ${ }^{(3)}$. A system using NAM (non-audible murmur) microphones was also reported by $\mathrm{S}$. Shimizu, et al. ${ }^{(4) 5)}$. NAM microphone was developed to detect a very weak speech sound. NAM microphone can be produced more cheaply than the electronic stethoscope, and has an advantage in commercialization. In recent years, development of microcomputers makes it possible easier and more cheaply to structure a measurement system than before. It is expected that a system which examines the health condition at a distant place, which diagnoses an internal disease are developed by combining the body conduction sound sensor with a microcomputer, and the system would be small and not get in the way of daily life.

In this study, we made a measurement system of body conduction sounds using a body conduction sound sensor, mbed LPC1768 ${ }^{(6)}$ and $\mathrm{XBee}^{(7)}$. A body conduction sound sensor using electret condenser microphone is filled up with polyurethane resin. The electret condenser microphone is also used on the NAM microphone. The mbed LPC1768 has a 32 bit microcontroller, it has more coping skills than the other 8 bit microcontrollers and it is good to deal with sound data. The system can communicate to a computer over a radio using XBee. It can filter out the noise using a median filter. We measured several body conduction sounds using this system at the cardiac apex, neck and wrist. We could measure coronary sounds at the cardiac apex well, the pulse waves at the neck and wrist well. Health conditions would be obtained from analysis of the measured sound. 


\section{Measurement System}

\subsection{Body conduction sound sensor}

The body conduction sound sensor is made by use of an improved small ECM (Primo Co., EM166). The microphone whose case is drilled a hole in and diaphragm is exposed is used. Figure 1 shows the structure of the sensor. The structure is based on the NAM microphone which is reported by S. Shimizu, et al.. The microphone is fixed on a vinyl chloride disk of $21 \mathrm{~mm}$ diameter, $2 \mathrm{~mm}$ thickness. An acrylic cylindrical case, $21 \mathrm{~mm}$ outer diameter, $17 \mathrm{~mm}$ inner diameter, $6 \mathrm{~mm}$ height, is also fixed on the disk. The sensor is filled up with polyurethane resin, then it can detect sounds inside of the body. Figure 2 is a picture of the sensor before filling up with polyurethane resin.

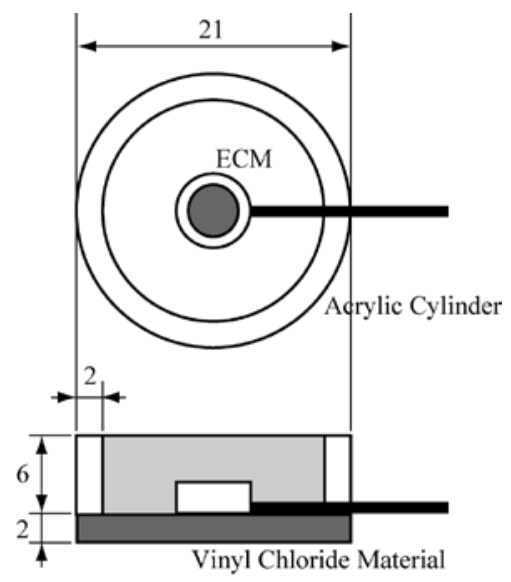

Fig. 1. Structure of the sensor.

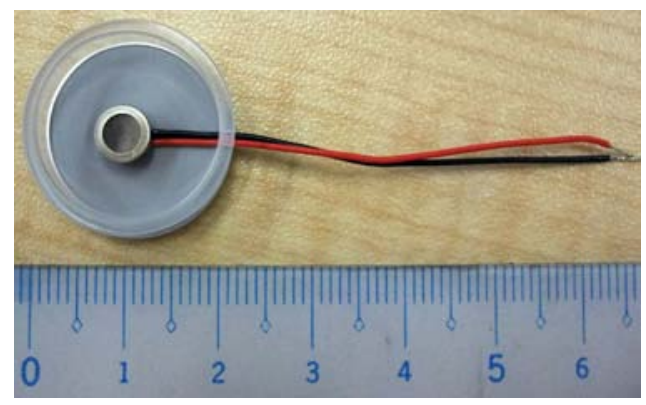

Fig. 2. The body conduction sound sensor before filling up with polyurethane resin.

\subsection{Mbed and XBee}

In this study, a mbed LPC1768 is used to control the measurement system. The mbed is an open-source electronics prototyping platform which consists of a 32 bit microcontroller on the board, a programming language and its development environment. Table 1 shows specifications of the mbed LPC1768 ${ }^{(6)}$. It is a microcontroller board based on the ARM Cortex-M3 microcontroller. It has 26 digital input-output pins and 6 analog inputs. It connects to a computer with a USB cable and we can write a program using the connection. Its development environment is provided on the Web. It doesn't require a special application to develop the mbed program, we can do well with a web-connected computer.

It has more coping skills than the other 8 bit microcontrollers. And the amount of memory installed is larger than the other 8 bit microcontrollers, it is good to deal with sound data. It has the flexibility of lots of peripheral interfaces, such as Ethernet and USB.

Table 1. Specifications of mbed LPC1768

\begin{tabular}{|c|c|}
\hline Core & ARM Cortex-M3 \\
\hline Frequency & $96 \mathrm{MHz}$ \\
\hline Flash Memory & $512 \mathrm{~KB}$ \\
\hline RAM & $32 \mathrm{~KB}$ \\
\hline Supply Voltage & $4.5-9.0 \mathrm{~V}$ \\
\hline Digital I/O Pins & 26 \\
\hline Analog Input Pins & 6 \\
\hline PWM Output Pins & 8 \\
\hline Analog Output Pins & 1 \\
\hline
\end{tabular}

It communicates to a computer over a radio using XBee module made by Digi International Inc.. XBee is a microcontroller that has a wireless communication function of ZigBee standards. Table 2 shows specifications of the XBee $802.15 .4^{(7)}$. Its frequency band is $2.4 \mathrm{GHz}$ and communication distance is dozens of meters. The power consumption is lower than the other wireless applications. Figure 3 is a picture of the measurement system we made and the device on the user's wrist.

Table 2. Specifications of XBee 802.15.4

\begin{tabular}{|c|c|}
\hline RF Data Rate & $250 \mathrm{kbps}$ \\
\hline Indoor/Urban Range \\
$\begin{array}{c}\text { Outdoor/ } \\
\text { RF Line-of-Sight Range }\end{array}$ & $100 \mathrm{~m}$ \\
\hline Transmit Power & $1 \mathrm{~mW}$ \\
\hline Frequency Band & $2.4 \mathrm{GHz}$ \\
\hline Serial Data Rate & $1200 \mathrm{bps}-250 \mathrm{kbps}$ \\
\hline Supply Voltage & $2.8-3.4 \mathrm{~V}$ \\
\hline Digital I/O Pins & 8 \\
\hline Analog Input Pins & 6 \\
\hline
\end{tabular}




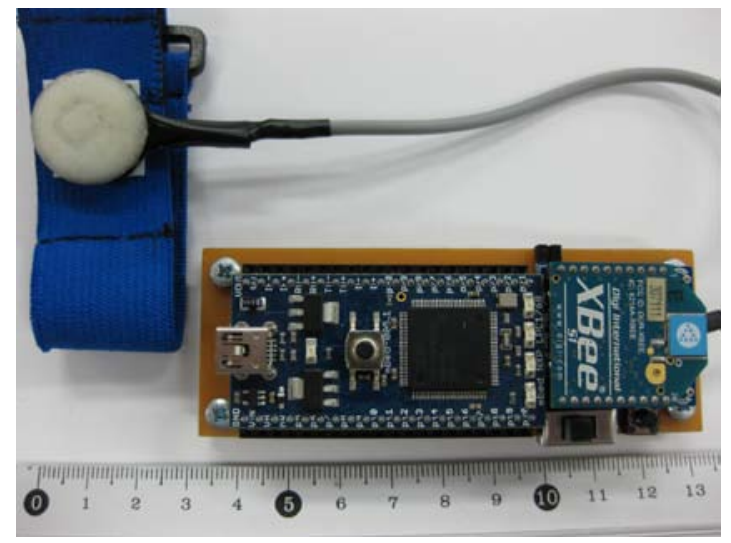

(a) Measurement system.

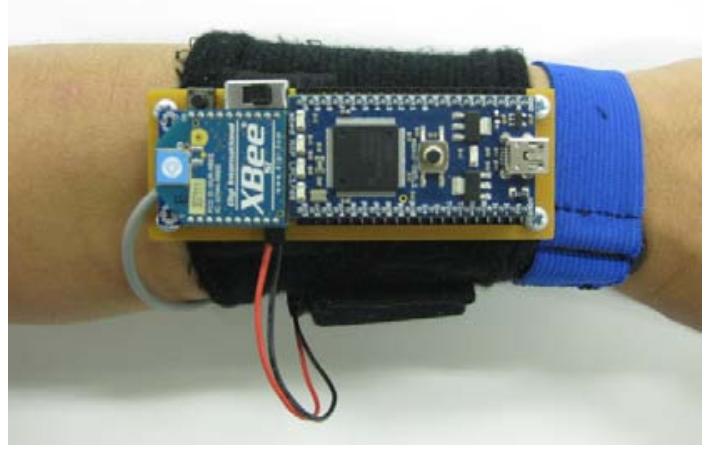

(b) The device on the user's wrist.

Fig. 3. Measurement system.

\subsection{Median filter}

To filter out the noise, we used median filter to the measured sound. The median filter gives a central value of three data that involve previous and next of each data. It is effective to filter out the noise of discontinuous change. Figure 4 shows a change in waveform using median filter.

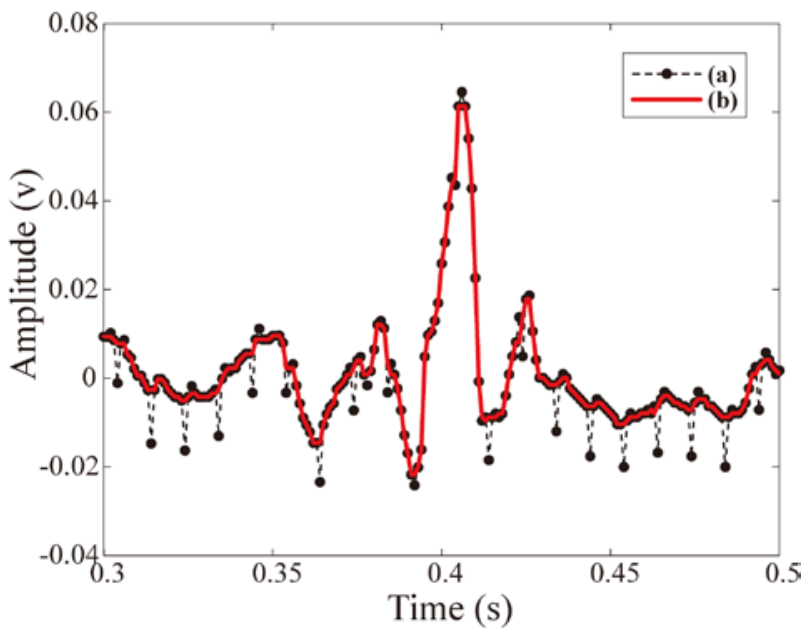

Fig. 4. Change in waveform using median filter.

(a) is measured sound. (b) is filtered the sound.

\section{Experiment}

To verify the effectiveness of this measurement system, we measured several body conduction sounds using this system. An examinee (Male, 40s) was healthy and physically unimpaired, kept in a seated position at rest. We measured three sounds at the cardiac apex, neck and wrist (Fig. 5). Sampling frequency is $1000 \mathrm{~Hz}$, measurement time is 5 seconds. Fig. 6 shows the measured sounds by the system. We could measure coronary sounds at the cardiac apex well, and could measure arterial sounds and the pulse waves at the neck and wrist well.

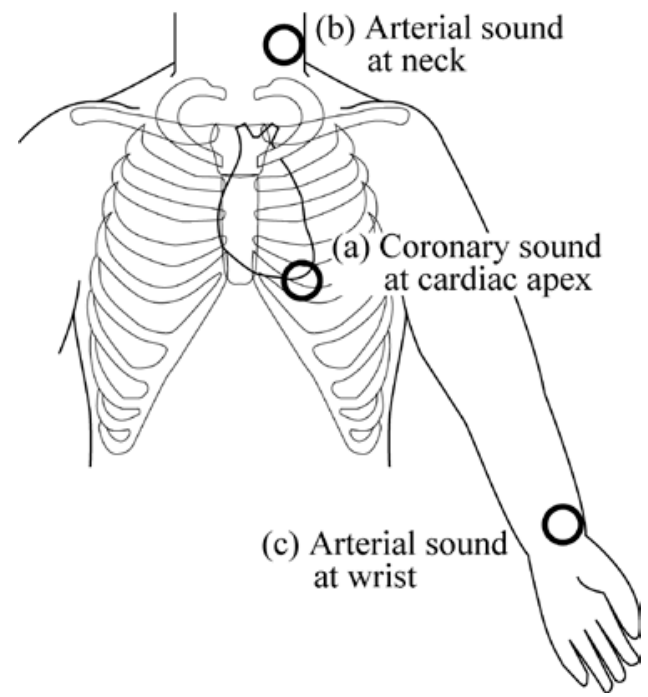

Fig. 5. Points of measurement.

\section{Conclusions}

In this study, we made a measurement system of body conduction sounds using a body conduction sound sensor, mbed LPC1768 and XBee. A body conduction sound sensor using electret condenser microphone is filled up with polyurethane resin. The mbed LPC1768 has a 32 bit microcontroller, it has more coping skills than the other 8 bit microcontrollers and it is good to deal with sound data. The system can communicate to a computer over a radio using XBee. It can filter out the noise using a median filter. We measured several body conduction sounds using this system at the cardiac apex, neck and wrist. We could measure coronary sounds at the cardiac apex well, and could measure arterial sounds and the pulse waves at the neck and wrist well. Health conditions would be obtained from analysis of the measured sound. 


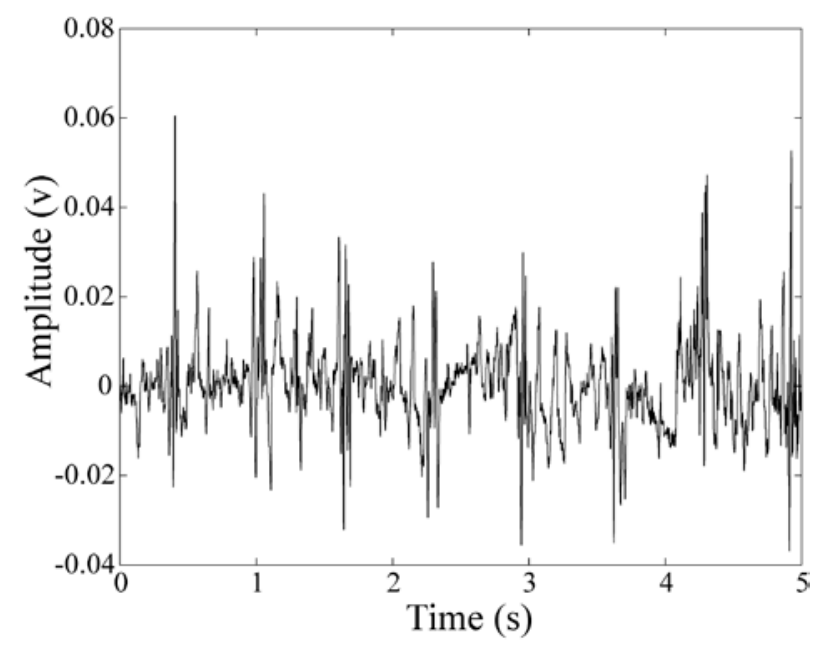

(a) Coronary sound at cardiac apex.

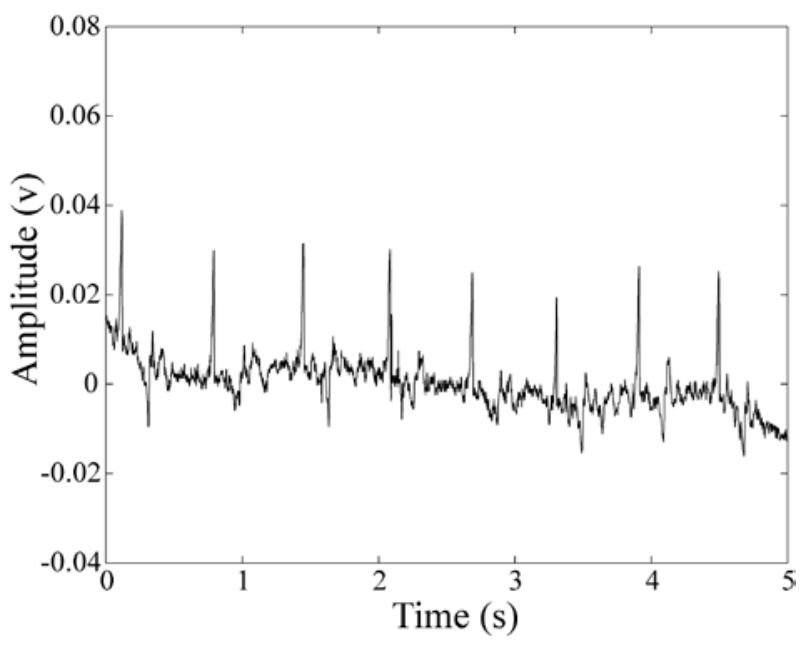

(b) Arterial sound at neck.

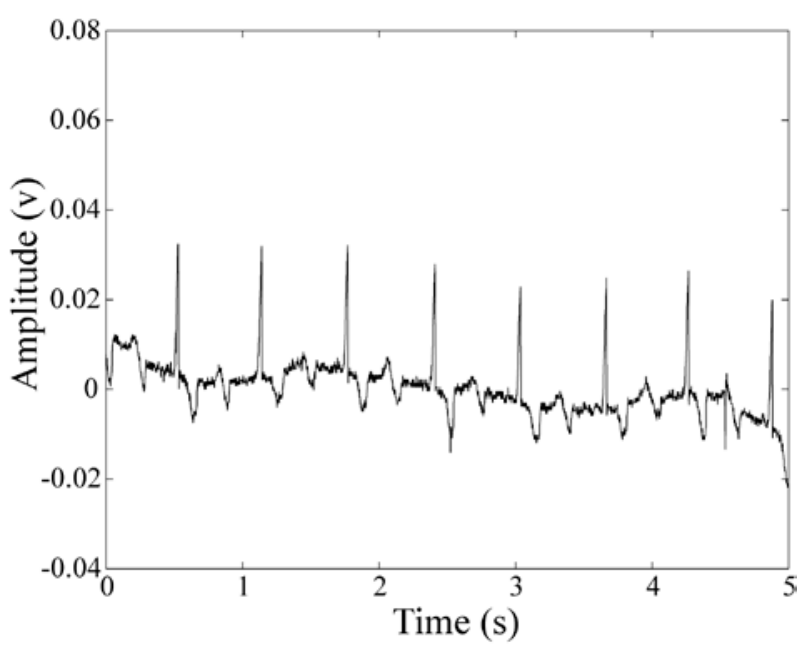

(c) Arterial sound at wrist.

Fig. 6. Measured sounds.

\section{References}

(1) J.R. Bulgrin, B.J. Rubal, C.R. Thompson and J.M. Moody : "Comparison of Short-Time Fourier, Wavelet and Time-Domain Analysis of Intracardiac Sounds", Biomed Sci. Instrum. Vol.29, pp.465-472, 1993

(2) C.H. Wu, C.W. Lo and J.F. Wang : "Computer-Aided Analysis and Classification of Heart Sounds Based on Neural Networks and Time Analysis”, Proc. IEEE Int. Conf. Acoust. Speech Signal Process, pp.355-3458, 1995

(3) Z. Jiang and S. Choi : "Development of Wireless Electronic Stethoscope System and Abnormal Cradiac Sound Analysis Method (Sound Characteristic Waveform Analysis)", The Japan Society of Mechanical Engineers, pp.140-147, 2005

(4) T. Hirahara and S. Shimizu : "Sensing various types sound generated inside the body using an ECM-based compact body-conducted sound sensor”, Proc. Autumn Meet. Acoust. Soc. Jpn., 2009

(5) S. Shimizu, M. Otani and T. Hirahara : "Frequency characteristics of several non-audible murmur (NAM) microphones”, Acoust. Sci. \& Tech., pp.139-142, 2009

(6) "Rapid Prototyping for Microcontrollers | mbed", http://mbed.org/, accessed December 28, 2012

(7) XBee RF Modules - Digi International”, Digi International Inc., http://www.digi.com/products/wireless-wired-embedde d-solutions/zigbee-rf-modules/zigbee-mesh-module/, accessed December 28, 2012 